\title{
Tentative detection of micron-sized forsterite grains in the proto-planetary disk surrounding HD 100453*
}

\author{
B. Vandenbussche ${ }^{1}$, C. Dominik ${ }^{2}$, M. $\mathrm{Min}^{2}$, R. van Boekel ${ }^{2,3}$, L. B. F. M. Waters ${ }^{1,2}$, G. Meeus ${ }^{4}$, and A. de Koter ${ }^{2}$ \\ 1 Instituut voor Sterrenkunde, Katholieke Universiteit Leuven, Celestijnenlaan 200B, 3001 Heverlee, Belgium \\ e-mail: bart . vandenbussche@ster. kuleuven. ac. be \\ 2 Astronomical Institute "Anton Pannekoek", University of Amsterdam, Kruislaan 403, 1098 SJ Amsterdam, The Netherlands \\ 3 European Southern Observatory, Karl-Schwarzschildstrasse 2, 85748 Garching bei München, Germany \\ 4 Astrophysikalisches Institut Potsdam, An der Sternwarte 16, 14482 Potsdam, Germany
}

Received 10 March 2003 / Accepted 6 July 2004

\begin{abstract}
We have re-analyzed the ISO-SWS spectrum of the intermediate mass pre-main-sequence star HD 100453. We confirm the weakness of the $10 \mu \mathrm{m}$ amorphous silicate band. We have found strong indications of the presence of a crystalline silicate emission at $34 \mu \mathrm{m}$, which had escaped detection so far due to severe glitches in the data. The 23.5 and $28.5 \mu \mathrm{m}$ forsterite bands have not been detected. This result indicates that the absence of crystalline silicate features at wavelengths shorter than $30 \mu \mathrm{m}$ does not prove the absence of crystalline silicate material in the circumstellar disks of young stars. The $34 \mu \mathrm{m}$ feature can be fitted well with crystalline forsterite grains with an average size of at least $2 \mu \mathrm{m}$, but the precise size is uncertain due to the poor data quality. The temperature of these grains must be $110 \mathrm{~K}$ or below, or otherwise the 23.5 and 28.5 micron features would be visible as well. Ground-based $10 \mu \mathrm{m}$ spectra show prominent emission from Polycyclic Aromatic Hydrocarbons and a weak silicate band whose shape suggests that it is dominated by a few micron size grains, of unknown lattice structure. The absence of any significant forsterite structure in the 10 micron region limits the mass of warm forsterite grains with sizes less than a few microns to $\leq 2 \times 10^{-4}$ of the mass of cold forsterite. Forsterite may be present in the warm regions, but then must be contained in even larger grains. The absence of $10 \mu \mathrm{m}$ silicate emission in some Herbig Ae stars is therefore due to the removal of small grains by dust growth processes.
\end{abstract}

Key words. stars: circumstellar matter - infrared: ISM

\section{Introduction}

It is by now well established that most young low- and intermediate mass stars have a circumstellar disk of gas and dust, which is the remnant of the accretion disk that formed the star (Mannings \& Sargent 1997; Natta et al. 2000). This disk has a life time of typically a few to 10 million years, and is believed to be the site of planet formation. The processes that govern the dissipation of the disk material and the formation of a planetary system are not well understood. Infrared imaging and spectroscopy is a useful tool to study these processes, since at these wavelengths changes in the composition of the dust can easily be established. The Infrared Space Observatory (ISO, Kessler et al. 1996) has revealed a rich mineralogy of oxygen-rich dust in a sample of bright Herbig Ae/Be stars, i.e. intermediate mass pre-main-sequence stars, pointing to

* Based on observations obtained at the European Southern Observatory (ESO), La Silla, and on observations with ISO, an ESA project with instruments funded by ESA Member States (especially the PI countries: France, Germany, The Netherlands and UK) and with the participation of ISAS and NASA. substantial grain processing in these disks (e.g. Malfait et al. 1998, 1999; Meeus et al. 2001; Bouwman et al. 2001; van Boekel et al. 2003). Here we report on a re-analysis of the ISOSWS spectrum of HD 100453, previously studied by Meeus et al. (2001) and Meeus et al. (2002). While in the earlier analysis no features were detected in the $30-40 \mu \mathrm{m}$ region, our reanalysis shows strong indications of the presence of a silicate band around $34 \mu \mathrm{m}$. At shorter wavelengths the spectrum lacks the forsterite (crystalline silicate) bands.

The paper is organized as follows: Sect. 2.1 discusses the new reduction of the ISO-SWS spectrum, and Sect. 2.2 presents a new ground-based $N$-band spectrum. We analyze and discuss the spectra in Sects. 3 and 4.

\section{Observations}

\subsection{New reduction of the ISO spectrum}

Following the tentative detection of a weak silicate feature in a ground-based $10 \mu \mathrm{m}$ spectrum (see Sect. 2.2), we re-analyzed the ISO-SWS spectrum (ISO observation 26000230) to investigate the presence of weak bands that may have escaped attention so far. We used the most recent calibration files and the 
interactive tools within the SWS Interactive Analysis System. We applied the standard reduction steps and calibration of the ISO-pipeline version 10, including the correction for transient effects in the signal of detector band 2 of the SWS.

The flux-calibrated signals of the individual detectors were then inspected for signals deviating significantly from the mean of all detectors. Deviations were seen on scales of the entire scan of a band (e.g. due to noise in the detector dark current measurements or the photometric calibration measurement of the internal calibration source) and on a local scale. On a local scale all detector bands occasionally suffer from sudden individual detector signal jumps, often attributed to cosmic ray hits of the read-out electronics. Local deviations in band 2 and especially band 4 are mainly caused by the impact of cosmic particles on the detector material. In the detector signal this is seen as a step function in the detector signal, followed by the so-called "glitch-tail": the transient effect after the signal glitch.

The SWS scans every spectral band twice: once from long to short wavelengths, and once from short to long wavelengths. Comparison of the two scans of the same wavelength region makes it possible to detect so-called "band-jumps" when all the detectors of a band exhibit a step function. After about one minute the signals have settled back at the nominal signal level. This effect is not well understood; therefore the most accurate correction is to discard the data points recorded for the entire detector band between the band jump and the moment when the signal settles at the nominal level. A major band jump occurs in band $3 \mathrm{a}$ at $137 \mathrm{~s}$ after the start of the observation. The signal decays for $50 \mathrm{~s}$. A smaller anomalous fluctuation occurs at $220 \mathrm{~s}$ for $25 \mathrm{~s}$. We have discarded the data points in the individual detector signals that suffered from the different effects described above.

Subsequently we applied a noise filter on the oversampled spectrum of every spectral band. Per resolution bin the data points that deviated by more than 2.5 times the standard deviation over the bin were discarded. This process was repeated five times. The spectrum was resampled to the same resolution bins, observing an oversampling factor of 3 to meet the Nyquist criterion of complete sampling. The resulting spectra of all bands were combined into one spectrum, which is shown together with model fits in Fig. 3.

The ISO-SWS spectrum of HD 100453 shows overall good agreement with that previously published by Meeus et al. (2001), with the exception of the 30-40 $\mu \mathrm{m}$ region ( $S W S$ band 4). We carefully re-inspected the individual detector scans in that wavelength range, in both scan directions, and detected an emission band around $34 \mu \mathrm{m}$ which had been hidden in the glitch-induced noise of the final spectrum in earlier reductions. Figure 1 shows the spectra from the two independent scans of the $30-45 \mu \mathrm{m}$ region. Most features in this wavelength range are not reproduced in both scans and are caused by the remnants of weaker signal glitches and subsequent transient effects which have not been discarded as described above. Around $34 \mu \mathrm{m}$ a broad feature appears in both independent scans. Between 30 and $36 \mu \mathrm{m}$ the signal-to-noise is higher, as the detector response is 40 percent higher than for instance at $42 \mu \mathrm{m}$. There are also no features in the Relative Spectral Response Function (RSRF) which could introduce false

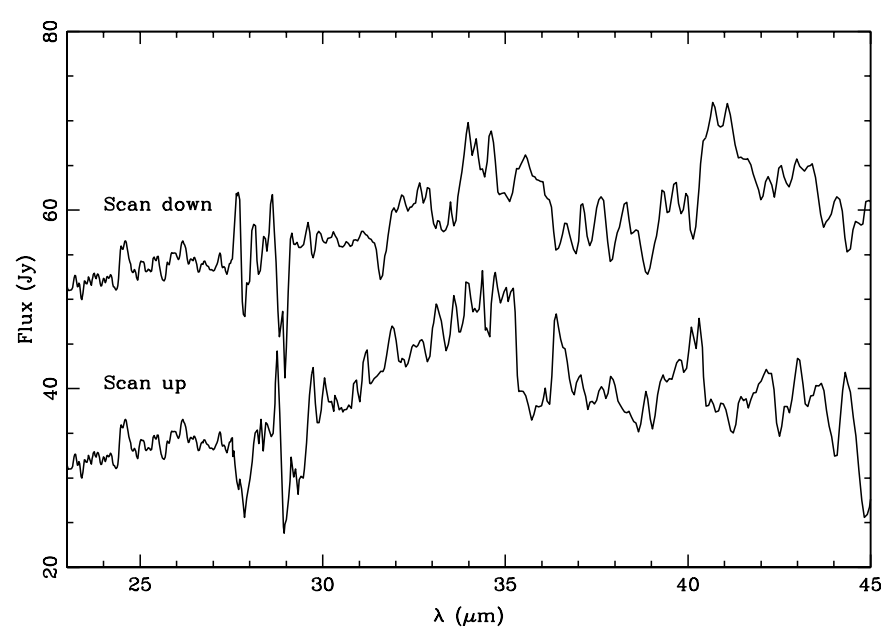

Fig. 1. The independent up and down scans of band 4 of the ISO-SWS spectrum of HD 100453. We have also added part of band 3 (the region between 23 and $27.6 \mu \mathrm{m}$, averaged between both scans) to show the continuum around the feature.

features with this shape, e.g. after an erroneous dark current subtraction. The two scans are performed in opposite directions, i.e. from long to short wavelengths and from short to long wavelengths, while the shape of the feature in the two scans is similar. Therefore it is not likely that the feature is an artifact of a transient effect. We conclude that, although the wavelength region between 30 and $45 \mu \mathrm{m}$ exhibits a very poor signal-to-noise, there are distinct indications of the presence of an emission band around $34 \mu \mathrm{m}$. The shape and the strength of the feature are uncertain at the $50 \%$ level.

\subsection{Ground-based N band spectrum}

The ground based spectrum was obtained on March 182003 using the Thermal Infrared Multi Mode Instrument 2 (TIMMI2, Reimann et al. 1998), mounted at the $3.60 \mathrm{~m}$ telescope at the ESO La Silla observatory. In order to correct for the strong atmospheric and instrumental background at $10 \mu \mathrm{m}$ we employed chopping and nodding, using a $+10^{\prime \prime}$ chop throw north-south, and $\mathrm{a}-10^{\prime \prime}$ nod throw north-south. The spectral resolution of the ground based spectrum is $R \approx 160$. Spectroscopic standard stars were observed regularly and were used to correct for the non-uniform atmospheric transmission.

In Fig. 2 we show the ISO-SWS spectrum and the ground based spectrum in the $10 \mu \mathrm{m}$ range. The two spectra agree very well, though the signal-to-noise ratio of the ground based spectrum is much higher. The ground based spectrum shows a weak, very flat emission band around $10 \mu \mathrm{m}$. Emission bands from Polycyclic Aromatic Hydrocarbons (PAHs) are clearly seen at 8.6 and $11.3 \mu \mathrm{m}$. Small forsterite grains could contribute to the $11.3 \mu \mathrm{m}$ emission, but would also create structure at $10 \mu \mathrm{m}$ which is not seen. Therefore we believe that the $11.3 \mu \mathrm{m}$ feature is entirely due to PAHs. The strong $9.7 \mu \mathrm{m}$ feature arising from small, amorphous silicates - observed in many Herbig Ae/Be stars - appears completely absent. 


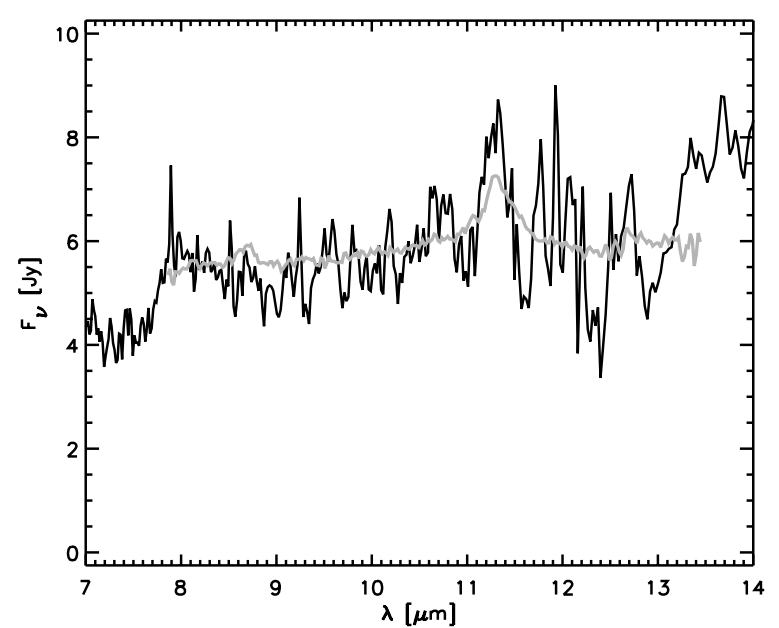

Fig. 2. The groundbased spectrum of the $10 \mu \mathrm{m}$ regions (grey line), compared with the ISO-SWS spectrum (black line).

\subsection{Summary of observational facts}

Before we analyze the spectrum in detail, we summarize the most important facts.

1. The newly reduced ISO spectrum shows a broad feature at $34 \mu \mathrm{m}$. Crystalline Forsterite has a feature at this wavelengths which is seen in many sources including other Herbig AeBe stars (Malfait et al. 1998). However, other features of forsterite at 19.7, 23.7 and $27.6 \mu \mathrm{m}$ are not seen.

2. The groundbased $N$-band spectrum shows no structure that could be attributed to small (crystalline or amporphous) silicate grains.

\section{Analysis of the spectrum}

For the analysis, we use a modified ISO spectrum where we replaced the region between 8 and $13.5 \mu \mathrm{m}$ by the higher signalto-noise ground based spectrum. The combined spectrum is shown in Fig. 3, together with model fits.

In order to study the infrared spectrum we have constructed a very simple model consisting of a warm and a cold dust component (see e.g. Bouwman et al. 2000). Meeus et al. (2001) argued that the spectral energy distribution of HD 100453 can be well explained by a warm optically thin, flared disk surrounding an optically thick, cold disk. Both components contain a mixture of carbon, amorphous olivine and forsterite at a single temperature. We fitted the size distributions of all dust materials and the temperatures of the cold and warm components.

We calculate the absorption cross sections of the dust grains from measurements of the bulk refractive index of the dust material. To do this we need to make an assumption about the grain shape and internal structure. The easiest approach is to assume that the grains are homogeneous and spherical. This gives reasonably good results for calculations of the emission efficiencies for amorphous grains. However, studies of the absorption spectra of small crystalline silicates have shown that calculations for homogeneous spherical particles do not agree well with measurements (e.g. Min et al. 2003; Hony et al. 2002; Bouwman et al. 2001). In order to model the

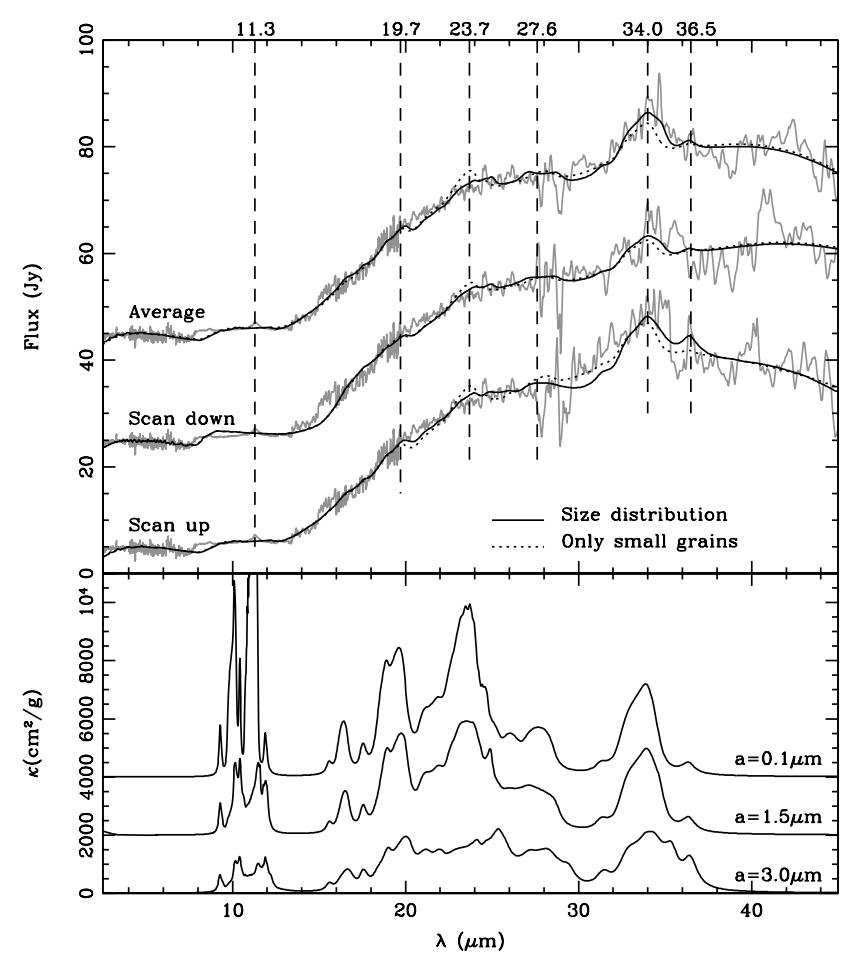

Fig. 3. Upper panel: fits to the spectra of HD 100453 for different scans. The observed spectra use the SWS spectrum except for the 10 micron region where the ground-based spectrum has been inserted. The 3 different spectra differ in which scan(s) hase been used in the SWS band 4. The vertical lines indicate the positions of important forsterite emission features. The full lines are the best fit to the spectra using a size distribution. Dotted lines indicate best fits using only small grains. The model parameters are listed in Table 1. Lower panel: absorption coefficients for crystalline forsterite grains with radius $0.1 \mu \mathrm{m}, 1.5 \mu \mathrm{m}$ and $3 \mu \mathrm{m}$, respectively. Note that all features except the $34 \mu \mathrm{m}$ feature are dramatically reduced when the grains reach a radius of $3 \mu \mathrm{m}$.

absorption properties of nonspherical grains, the most common approach is to use a continuous distribution of ellipsoids (Bohren \& Huffman 1983) which has been applied to measured optical constants of crystalline Forsterite for example by Fabian et al. (2001). However, it is important to notice (and often ignored) that the CDE approximation is only applicable to particles in the Rayleigh limit, which requires $|m| \frac{2 \pi a}{\lambda} \ll 1$ where $|m|$ is the absolute value of the complex refractory index of the material. Therefore, the size of particles which may be considered to be in the Rayleigh limit depends upon the strength of the considered resonance. For the strong resonances of forsterite, $|m|$ can reach values of 10 . In order to calculate the absorption properties of grains at the $34 \mu \mathrm{m}$ feature of Forsterite, CDE is only applicable for particles much smaller than $0.5 \mu \mathrm{m}$ ! Min et al. (2003) have shown that excellent agreement between calculations and measurements of forsterite grains can be obtained by using the so-called statistical approach. In this approach the absorption properties of an ensemble of irregular particles is simulated by the average absorption properties of an ensemble of various simple shapes. We use here a distribution of hollow spherical particles for the forsterite grains which is in excellent agreement with the positions and strengths of forsterite 
resonances obtained from laboratory measurements. Note that we do not claim that the grains we observe are hollow spheres. Rather, a distribution of hollow spheres with varying inner hole size can be used as a calculation method to derive the optical properties of irregular grains, also outside the Rayleigh limit. We average over the fraction of the total volume occupied by the central inclusion, $f$, over the range $0 \leq f<1$. All particles in the distribution have the same material volume, so the particles with higher inclusion fraction have a larger outer radius. For details of this distribution see Min et al. (2003). The hollow spherical particles are calculated using a simple extension of Mie theory (Bohren \& Huffman 1983) allowing us to calculate the emission efficiencies for all possible grain sizes. The bulk refractive index as a function of wavelength for carbon was taken from Preibisch et al. (1993), for amorphous olivine from Dorschner et al. (1995) and for forsterite from Servoin \& Piriou (1973). For the carbon and amorphous olivine grains we use homogeneous spheres.

The results of the opacity calculations for forsterite grains are shown in the lower panel of Fig. 3 where we plot the mass absorption coefficient calculated for grains with different radii. One clearly sees that increasing the size of the grains leads to an almost complete disappearance of the 19.7, 23.7 and $27.8 \mu \mathrm{m}$ features, while the strength of the $34 \mu \mathrm{m}$ feature is only reduced slightly. However, even the $3 \mu \mathrm{m}$ grains still exhibit some structure in the $10 \mu \mathrm{m}$ region, albeit much weaker than sub-micron grains.

For the grain size distribution we use a power law given by

$n(a)= \begin{cases}c \cdot a^{\alpha}, & a_{\min } \leq a \leq a_{\max }, \\ 0, & \text { elsewhere. }\end{cases}$

Here $a$ is the radius of a sphere with the same material volume as the dust grain, $a_{\min }$ and $a_{\max }$ are the minimum and maximum grain sizes and $\alpha$ is the slope of the power law. We have no detailed information on the size distributions of the carbon and amorphous olivine grains since they contribute only to the continuum flux. The resulting fit parameters of these dust components will therefore not be discussed any further.

We fit the spectrum by maximizing $1 / \chi^{2}$, where

$\chi^{2}=\int_{\lambda_{\min }}^{\lambda_{\max }}\left|\frac{F_{\text {obs }}(\lambda)-F_{\text {model }}(\lambda)}{\sqrt{F_{\text {obs }}(\lambda)}}\right|^{2} \mathrm{~d} \lambda$.

Here $F_{\text {obs }}$ is the observed flux and $F_{\text {model }}$ is the modeled flux. We maximize $1 / \chi^{2}$ as a function of the grain size distribution parameters and the dust temperatures of the cold and warm component. We use a genetic optimization algorithm called PIKAIA (Charbonneau 1995).

Figure 3 shows the resulting best model fits. In order to get a handle on the uncertainties of the results, we have fitted both spectral scans of band 4 independently as well as the average spectrum. The fit parameters are listed in Table 1. It is apparent that the $34.0 \mu \mathrm{m}$ feature is present in all scans. The upscan seems to show an indication of the $36.5 \mu \mathrm{m}$ feature, but this is not visible in the downscan. At shorter wavelengths, there is very little evidence for any of the features at 27.6, 23.7, or $19.7 \mu \mathrm{m}$. The resulting fit parameters strongly favor large grains. In Table 1 we also present the mass-averaged radius
Table 1. Model parameters for fitting the spectrum of HD 100453.

\begin{tabular}{lcccc}
\hline \hline Scan & $\begin{array}{c}\text { Size range } \\
\mu \mathrm{m}\end{array}$ & $\alpha$ & $\begin{array}{c}\text { Mass avg. } \\
\text { radius }(\mu \mathrm{m})\end{array}$ & $\begin{array}{c}\text { Warm/Cold forsterite } \\
\text { mass ratio }\end{array}$ \\
\hline Average & $1.5-1.9$ & -2.4 & 1.7 & $1.2 \times 10^{-4}$ \\
Scan 1 (down) & $1.2-1.9$ & -4.0 & 1.5 & 0.0 \\
Scan 2 (up) & $0.1-8.0$ & -3.0 & 4.1 & $4.2 \times 10^{-5}$ \\
\hline
\end{tabular}

(which is the average radius of the particles weighted by their mass) for the three cases. This shows that the distributions of the dust mass are heavily biased toward grains with radii of several $\mu \mathrm{m}$. If we take the average scan as representative, most of the material is present as $1.7 \mu \mathrm{m}$ grains. Another way to show that emission is dominated by these grains is comparing with a fit involving only submicron grains. Such fits for all three cases are indicated by the dotted lines in Fig. 3. The best fits with small grains clearly show a weak feature at $23.7 \mu \mathrm{m}$ (and also an indication for a feature at $27.6 \mu \mathrm{m}$ ). These are not seen in the data. The peak position for the 34.0 micron feature shifts to $33.6 \mu \mathrm{m}$ for small grains, less consistent with the observations than the large grain fit.

We have used the same size distribution for the cold and warm components. The grain sizes are mostly constrained in the cold component. The lack of structure due to forsterite in the $10 \mu \mathrm{m}$ region imposes that at most about $7 \times 10^{-4}$ of the mass in the forsterite grains can be cold forsterite.

\section{Discussion}

The absence of a strong $10 \mu \mathrm{m}$ silicate emission feature in the spectrum of HD 100453 and several other Herbig Ae stars has been a puzzle for quite some time (Meeus et al. 2001). In a recent study, Meeus et al. (2002) investigated two possible causes for this observation. Since the emission feature is due to small warm silicate grains, the absence of an emission feature must mean that no such grains are present. Either all small grains have been converted to large grains by coagulation, or the geometry of the disk causes any small silicate grains to be too cold to contribute in the 10 micron region. The geometric option was based on models of passive disks with an inner hole and puffed-up inner rim (Dullemond et al. 2001). In these models, the rim casts a shadow and reduces the temperature of the material outside of the rim in a region between about 1 and $7 \mathrm{AU}$. However, in the mean time it has been shown that the shadowing of the inner rim is not efficient enough to suppress the emission of a $10 \mu \mathrm{m}$ feature (Dullemond \& Dominik 2004). While shadowing reduces the temperature of grains in the shadow, the temperature stratification in that region remains such that an emission feature is still produced and of similar strength compared to unshadowed disks. This seems to rule out shadowing as the cause of the weakness of the $10 \mu \mathrm{m}$ feature.

We conclude that small grains must have been efficiently removed. This indicates that grain growth taking place in the disk is responsible for the observed lack of a $10 \mu \mathrm{m}$ feature. The current study presents further evidence in this direction.

The most interesting result is that these grains must be large and cold at the same time. We have unsuccessfully tried to fit 
the entire spectrum with a passive disk model (Dullemond et al. 2001) using a single grain composition. Any grain composition able to fit the $34.0 \mu \mathrm{m}$ feature also produced structure in crystalline features at shorter wavelengths. Therefore, the grain composition in HD 100453 must depend on distance from the star with forsterite grains in the warm component being either less abundant or much larger in size than those present in the cold component. Even $3 \mu \mathrm{m}$ grains still produce structure in the $10 \mu \mathrm{m}$ region, and grains smaller than the derived size of at least $2 \mu \mathrm{m}$ would produce detectable features at 19.7, 23.7 and $27.6 \mu \mathrm{m}$. Any crystalline grains in the inner disk regions would therefore have to be considerably larger.

Upcoming observations with the Spitzer space telescope will undoubtedly show the $30-40 \mu \mathrm{m}$ region with much better signal-to-noise. This independent observation is indispensable for having final evidence about the presence, strength and position of the $34 \mu \mathrm{m}$ emission feature. Given the strong dependence of feature shape and position on grain size, such observations will make it possible to measure the size of the crystalline forsterite grain in this source very accurately. In the future, spatially resolved observations of the mineralogy of this source are bound to yield more surprises.

The tentative detection of the $34 \mu \mathrm{m}$ feature in the ISO-SWS spectrum of HD 100453 also shows that the absence of crystalline silicate features at wavelengths shorter than $30 \mu \mathrm{m}$ does not conclusively show that no crystalline silicate material is present at all.

\section{Summary}

A re-analysis of the ISO spectrum of HD 100453 has given strong indications of the presence of an emission band in the $34 \mu \mathrm{m}$ region. This feature can be attributed to crystalline forsterite grains with a size of $\sim 2 \mu \mathrm{m}$. Therefore, the cold dust in the disk around HD 100453 is highly processed. Grains have grown by coagulation, and have been annealed. In the warm component, any crystalline grains need to be either less abundant or larger (than $2 \mu \mathrm{m}$ ) to be consistent with observations. The new results strongly suggests that the absence of a strong $10 \mu \mathrm{m}$ silicate emission feature in several Herbig Ae stellar disks is due to dust growth which has efficiently removed submicron grains. The presence of crystalline grains in the cold part of the disk has important consequences for models of radial mixing and/or local heating events in disks.

Acknowledgements. G.M. acknowledges financial support by the ECRTN on "The Formation and Evolution of Young Stellar Clusters" (RTN-1999-00436, HPRN-CT-2000-00155).

\section{References}

Bohren, C. F., \& Huffman, D. R. 1983, Absorption and Scattering of Light by Small Particles (New York: Wiley)

Bouwman, J., de Koter, A., van den Ancker, M. E., \& Waters, L. 2000, A\&A, 360,213

Bouwman, J., Meeus, G., de Koter, A., et al. 2001, A\&A, 375, 950

Bouwman, J., Meeus, G., de Koter, A., et al. 2001, A\&A, 375, 950

Charbonneau, P. 1995, ApJS, 101, 309

Dorschner, J., Begemann, B., Henning, T., Jäger, C., \& Mutschke, H. 1995, A\&A, 300, 503

Dullemond, C., \& Dominik, C. 2004, A\&A, 417,159

Dullemond, C. P., Dominik, C., \& Natta, A. 2001, ApJ, 560, 957

Fabian, D., Henning, T., Jäger, C., et al. 2001, A\&A, 378, 228

Hony, S., Waters, L. B. F. M., \& Tielens, A. G. G. M. 2002, A\&A, 390,533

Kessler, M. F., Steinz, J. A., Anderegg, M. E., et al. 1996, A\&A, 315, L27

Malfait, K., Waelkens, C., Bouwman, J., de Koter, A., \& Waters, L. B. F. M. 1999, A\&A, 345, 181

Malfait, K., Waelkens, C., Waters, L. B. F. M., et al. 1998, A\&A, 332, L25

Mannings, V., \& Sargent, A. I. 1997, ApJ, 490, 792

Meeus, G., Bouwman, J., Dominik, C., Waters, L. B. F. M., \& de Koter, A. 2002, A\&A, 392, 1039

Meeus, G., Waters, L. B. F. M., Bouwman, J., et al. 2001, A\&A, 365, 476

Min, M., Hovenier, J. W., \& de Koter, A. 2003, A\&A, 404, 35

Natta, A., Grinin, V., \& Mannings, V. 2000, Protostars and Planets IV, 559

Preibisch, T., Ossenkopf, V., Yorke, H. W., \& Henning, T. 1993, A\&A, 279, 577

Reimann, H., Weinert, U., \& Wagner, S. 1998, in Proc. SPIE, ed. Albert M. Fowler, Infrared Astronomical Instrumentation, 3354, 865

Servoin, J. L., \& Piriou, B. 1973, Phys. Stat. Sol. (b), 55, 677

van Boekel, R., Waters, L. B. F. M., Dominik, C., et al. 2003, A\&A, 400, L21 